\title{
ATRACCIÓN DE INVERSIÓN EXTRANJERA DIRECTA EN MÉXICO: EL EFECTO DEL NIVEL EDUCATIVO
}

Beatriz Rosas Rodríguez*

\begin{abstract}
Rosas-Rodríguez B. Atracción de inversión extranjera directa en México: El efecto del nivel educativo. Hitos de Ciencias Económico Administrativas 2016; 22 (62): 27-36
\end{abstract}

RESUMEN

Objetivo: Conocer cómo afecta el nivel educativo a la atracción de Inversión Extranjera Directa, dadas las demás variables independientes de control que explican la IED.

Material y método: Esta investigación es cuantitativa, experimental de serie cronológica, debido a que se analizarán las observaciones de cada una de las variables durante un periodo de tiempo, que comprende desde 1994 a 2013. Todos los datos obtenidos de las variables serán a nivel nacional. Tomando en cuenta los factores que inciden en el entorno nacional que pueden tener efecto sobre las decisiones de inversión de otros países hacia México, como: el ambiente económico, la infraestructura de transporte y de telecomunicaciones y la seguridad pública.

Resultados: Con base en los resultados, un mayor nivel educativo afecta negativamente la inversión extranjera directa. A mayor graduados de un nivel educativo superior, menos inversión extranjera directa; debido a que las empresas
Rosas-Rodríguez B. Attracting foreign direct investment in Mexico: The effect of the level educational. Hitos de Ciencias Económico Administrativas 2016; 22 (62): 27-36

ABSTRACT

Objetive: Know how educational level affects to foreign direct investment attraction, given others independent control variables that explains FDI.

Material and method: This research is quantitative, experimental and time's series, due to the observation are analyzed for a time period which ranges from 1994 to 2013. All data were obtained from nationwide variables. Based on factors that influence in national environment and may have effect on investment decisions from other countries towards Mexico as: economic environment, transportation and telecommunication infraestructure and public security.

Results: Based on the outcomes, a higher education level affects in a negative way foreign direct investment. To more graduates from higher education level, less foreign direct investment, due to multinational companies require a greater

\footnotetext{
*Estudiante e investigadora de la Maestría en Ciencias Económico Administrativas. Universidad Autónoma de Querétaro. Facultad de Contaduría y Administración.
} 
multinacionales requieren una mayor cantidad de mano de obra con pocas habilidades y formación.

Palabras clave: Inversión Extranjera directa. Nivel educativo. Infraestructura de transporte y telecomunicaciones. Seguridad social. Empleo. number of labor force with few skills and formation.

Key words: Foreing Direct Investment. Education level. Transportation and communication infrastructure. Public Security. Employment.

DIRECCIÓN PARA RECIBIR CORRESPONDENCIA: correo electrónico: beatrizrosas@outlook.com

esde años previos a la firma del Tratado de Libre Comercio (TLC), «México ya implementaba ciertas políticas que lentamente lo iban llevando a una liberación económica cuyo primer objetivo era establecer relaciones económicas con otros países, a través de la firma de tratados» (De la Cruz Gallegos, Canfield Rivera, y González Castro, 2009, p. 18).

Un hecho importante que marcó el destino de la industrialización en México fue el Programa de Industrialización Fronteriza, creado en 1965, las decisiones en México fueron diferentes con respecto a las adoptadas en el este y sureste de Asia, para México el propósito de atracción de IED fue la reducción de las tasas de desempleo; por lo tanto, la evolución tecnológica en México ha sido menos exitosa (Dutrénit y Vera-Cruz, 2005, p. 2).

Se cree que la Inversión Extranjera Directa (IED) es beneficiosa para el crecimiento económico de un país; por ejemplo, Kumaran (2008), considera que «la IED comúnmente resulta en el incremento de la inversión nacional, incrementa la competencia y el acceso a los mercados de exportación» (pp. 135-136); pero, si dentro de esta afirmación se toman en cuenta otro tipos de factores de control que influyen en el crecimiento de un país, es posible que no exista una relación directa. En su investigación, Neycheva (2013) sugiere que un aumento de la proporción de personas con educación media superior en la mano de obra no está relacionada con la tasa de crecimiento de largo plazo (p. 332).

Este documento pretende conocer en qué sentido impacta el nivel educativo de la población económicamente activa a la IED, así como ofrecer una explicación alternativa al desempleo en cierta población en México. La información sobre el monto de IED que entra en un país de manera anual, permite conocer qué tan atractivo es ese país para los extranjeros.

Para Kato-Vidal (2013) «la teoría predice que el producto interno bruto puede crecer sólo si hay crecimiento de los factores productivos, incluido el nivel de la tecnología» (p. 213). Para el crecimiento de nivel de tecnología se requiere que las Empresas Multinacionales (EMN), instruyan al capital humano del país anfitrión.

Algo importante al momento de examinar los beneficios de la llegada de capital extranjero a territorio nacional, es el beneficio tecnológico que este capital podría traer al país, por medio de la transferencia de tecnología. «La transferencia de tecnología puede verse apoyada al estimular la capacitación y entrenamiento del capital humano, por medio de extranjeros y sugiere que el conocimiento dinamiza las economías» (Moreno Monsalve y Méndez Peón, 2014, p. 2).

Un dato relacionado con los beneficios que un país obtiene a través de la IED es que de acuerdo con la Organización para la Cooperación y desarrollo económico (OECD, 2013) los mexicanos más jóvenes tienen un nivel educativo 
más alto que los adultos, pero aun así, los más jóvenes son más vulnerables al desempleo; adicionalmente, las tasas de desempleo en México tienden a estar arriba de las tasas promedio.

Una respuesta rápida a la pregunta relacionada con las altas tasas de desempleo en países con IED la plantean Young y Lan (1997) ya que a fin de cuentas «la transferencia de tecnológica toma un lugar bastante modesto y se limita principalmente a los beneficios asociados con la producción extranjera, caracterizada por el trasplante de hardware y la formación básica de los trabajadores» (p. 676).

La hipótesis de esta investigación es que el nivel de estudios está inversamente relacionado con la atracción de inversión extranjera directa, esto quiere decir que a medida que aumenta el nivel de estudios de las personas, menor será la atracción de IED. Esto debido a que ciertas EMN que se instalan en México no consideran necesario el uso de capital humano con una gran cantidad de habilidades y conocimientos porque no se pretende que los trabajadores, conozcan y adopten la tecnología de las EMN.

\section{MARCO TEÓRICO}

La definición de IED para Molina-Martínez y Alcaraz-Vargas (2012), consiste en la inversión realizada para permitir los intereses de negocio de un inversor en una empresa que se encuentra ubicada en un país diferente, distinta al país de origen del inversor (p. 59). La IED se puede clasificar en dos tipos: interna y externa, la primera es cuando el capital extranjero se produce en recursos locales y la segunda, cuando el capital local se invierte en recursos extranjeros.

De acuerdo a (Kunhardt y Gutiérrez-Haces, 2013), «En la década que comprende los años de 1984 a 1994 los flujos de IED pasaron de un promedio de 248 millones de dólares, a un promedio 1.9 billones de dólares en la década 1995-2005» (p. 36).

Recordando que el punto de partida de la investigación es la IED en México y que parte de esta inversión se considera beneficiosa para el país, debido a su influencia en el crecimiento, esto permite la entrada de la variable educación dentro de esta investigación.

La atracción de la IED permite la entrada a un país de diversas empresas provenientes del extranjero, las cuales buscan producir en ese país, en su mayoría con los factores de producción que el país ofrece. Si la educación es uno de los determinantes de la IED, qué tipo de educación requieren las EMN.

Para Young y Lan et al. (1997), desde hace tiempo se reconoce que:

[...] la preocupación de la empresa en el establecimiento de operaciones en un país en desarrollo en particular no es promover el desarrollo de esa economía, sino para obtener beneficios satisfactorios. La teoría de la competencia tecnológica sugiere que el impacto de la tecnología extranjera en el desarrollo local depende del nivel de competencia tecnológica nacional. (p. 670).

Por lo tanto, Dutrénit y Vera-Cruz et al. (2005), comentan que al localizar a las maquilas inicialmente en las localidades en la frontera norte, donde existe una tradición basada en poca fabricación, un sistema educativo joven, inexistentes centros de investigación y desarrollo y una estructura institucional local inmaduro ( $p$. 2), no es sorprendente que no fuera una buena decisión estratégica, ya que estas maquiladoras eran las pioneras de la IED en México.

En un estudio realizado por Jordaan (2008), sus resultados sugieren que «la mejora del nivel educativo de la fuerza laboral de la región... puede aumentar la probabilidad de que una región sea 
seleccionada por las nuevas empresas de propiedad extranjera» (p. 408).

De acuerdo con Ramos (2015), México es parte de una de las regiones en las que un mayor número de empresas informan que tienen dificultades para encontrar las habilidades que necesitan y donde, a su vez, la inversión de las empresas en sus trabajadores está por debajo del promedio de la OCDE (p. 1).

Es por eso que, la educación juega un papel clave en el futuro de las economías modernas. La inversión eficaz y eficiente en el capital humano de una economía hace una contribución significativa al aumento de la competitividad y también puede salvaguardar la prosperidad de los ciudadanos individuales (Spieb, 2013, p. 3).

Una de las explicaciones a la casi inexistente transferencia de tecnología en algunas industrias mexicanas es que la transferencia de tecnología trae del extranjero con ella la posibilidad de la disipación de conocimientos y el fomento de la competencia (Young y Lan, 1997, p. 676).

Los trabajadores que se incorporan al mercado de trabajo rara vez se conectan con las actividades de alta tecnología, esto se debe al bajo nivel de innovación que existe en la economía mexicana. Como Ramos menciona «además de la reforma educativa, es necesario revisar las políticas que impactan en el deterioro de las habilidades de los trabajadores poco cualificados, que se emplean en trabajos precarios donde no tienen la oportunidad de recibir capacitación» ( $p$. 1).

En México lo datos de la (OECD, 2013), muestran que el $18.9 \%$ de los adultos jóvenes con educación media superior, y el $16.8 \%$ de los adultos jóvenes con la educación superior no son empleados ni se encuentran en formación profesional.
Por otro lado, si las políticas de atracción IED en México se han enfocado en la reducción del desempleo, es razonable la afirmación de que la inversión extranjera directa tiene efectos relativamente favorables de empleo, en particular para los trabajadores menos cualificados, en la producción de materiales y accesorios eléctricos (Waldrich, p. 1180).

Nuñez y Livanos (2010), afirman que un hecho establecido es que un alto nivel educativo está asociado con altos niveles de desempleo, debido a que quienes obtuvieron un grado académico, han tenido mayores tasas de desempleo que aquellos con nivel educativo menor (p. 475). Por lo tanto, ellos incluyen la variable desempleo asociándola con un grado académico mayor.

Además, documentos de la OECD (2013), explican que las tasas del empleo para las personas con logro menor a la educación media superior tienden estar por encima de la media de la OECD, y por debajo de la media en los niveles de educación más altos [...] la educación media superior y superior tienen las tasas de desempleo más altas, incluso si más jóvenes mexicanos tienen mayor nivel educativo, son más vulnerables al desempleo (OECD et al., 2013).

Adicionalmente, en su último informe anual de estadísticas educativas la OECD (2014) comenta: en México, los jóvenes de 15 a 29 años pasarán en promedio 6.4 años en actividades laborales y 5.3 años en educación y formación; un año más en el trabajo que el promedio de la OCDE (5.4 años) y dos años menos en educación (el promedio de la OCDE es de 7.3 años). Existe un gran riesgo de que estos se desvinculen tanto de la educación como del mercado laboral. Aun cuando la proporción de jóvenes de entre 15 y 29 años que no cursan estudios ha disminuido en comparación con el año anterior (OECD, 2014, p. 2). 
Complementando esta idea, Waldkirch, Nunnenkamp, y Alatorre Bremont et al. (2009), sugieren que la IED tiene un modesto impacto sobre el empleo y que en las industrias de capital intensivo el efecto solo es positivo para los trabajadores de cuello azul.

Sustentando las afirmaciones de que el nivel educativo no ayuda a obtener empleo:

Las tasas de desempleo más altas en México se encuentran en los niveles educativos más altos y éstas han aumentado en años recientes. [...] Suponiendo que el nivel de instrucción de la mano de obra refleja su nivel de capacitación, y que sean las empresas y ramas más dinámicas en la economía mexicana las que absorben mano de obra más calificada, resulta extraño que la desocupación general sea mayor a mayor nivel de capacitación (Ruiz Nápoles y Ordaz Díaz, 2013, p. 97).

Esto quiere decir, que los desocupados son en su mayoría aquellos con un mayor grado de instrucción y la tendencia está en aumento. A propósito Keller (2005), comenta que en México tener un mayor nivel de educación no se traduce necesariamente en un menor riesgo de desempleo. Al contrario de la mayoría de los países de la OCDE, donde tener un título universitario significa mejores posibilidades de encontrar calidad, empleos bien remunerados. [...] A la vista de los escasos recursos, aparecen los recursos públicos asignados a una mejor educación básica en lugar de superior las tasas de matriculación secundaria parecen los más vitales para el crecimiento per cápita (Keller, 2005, pp. 31-33).

La fuerza laboral bien educada es esencial para una economía innovadora, estas competencias incluyen conocimientos especializados, como muchas habilidades relacionadas con la innovación se desarrollan desde una edad temprana, tienen que ser adquiridos en parte a través de la educación formal (Ramos et al.,
2015, p. 9) y sugiere «orientación de estudiantes hacia la ciencia, la tecnología y las matemáticas» (p. 10).

\section{METODOLOGÍA}

Con base en la hipótesis que se mencionó anteriormente la cual sugiere que existe una relación inversa entre el nivel educativo y la IED, dado que a mayor nivel educativo menor será la empleabilidad de las personas en los puestos requeridos por las EMN que atraen IED al país, y en la revisión de la literatura. Este estudio analiza el efecto de algunos factores sobre la inversión extranjera directa.

Este documento difiere de otros que se hayan realizado, debido a que se centra en el efecto de la variable nivel educativo sobre la IED, utilizando adicionalmente otras variables de control; lo cual quiere decir que el principal objetivo es conocer cómo afecta el nivel educativo a la atracción de IED, dadas las demás variables independientes que explican la IED, interactuando conjuntamente.

Esta investigación es cuantitativa, experimental, de serie cronológica, debido a que se analizarán las observaciones que cada una de las variables durante un periodo de tiempo, que comprende desde 1994 a 2013. Toda la información obtenida de las variables será a nivel nacional.

Para realizar este análisis, se tomaron en cuenta factores que incurren en el entorno nacional que pueden tener efecto sobre las decisiones de inversión de otros países hacia México. Estos factores son: El ambiente económico se midió a través del nivel de inflación y el ingreso per cápita. El ambiente de infraestructura se midió por medio de la infraestructura de transporte y telecomunicaciones. El ambiente social se midió a través de la seguridad pública.

La base de datos correspondiente a la IED se obtuvo del Centro de Estudios de Finanzas Públicas (CEFP) de la Cámara de Diputados. Para 
obtener el nivel de inflación y el ingreso per cápita en México se utilizó la información del Banco Mundial.

Para medir la inversión en infraestructura en transporte que puede influir en decisiones de inversión se utilizó la información de la Secretaría de Comunicaciones y Transportes, específicamente del informe de las Principales Estadísticas de Comunicaciones y Transportes, correspondientes a la inversión pública y privada para la infraestructura carretera e inversión pública y privada para la infraestructura portuaria todas en millones de pesos. Para la variable que mide la infraestructura en telecomunicaciones, se utilizó como indicador el número de suscripciones a teléfonos fijos, obtenida también de la Secretaría de Comunicaciones y Transportes.

La seguridad pública se midió por medio de los presuntos delincuentes y delincuentes sentenciados por fuero federal y fueron común, (mismos que hasta el año 2008 se les nombró como presuntos delincuentes y a partir del 2009 se les conoce como procesados, mientras los delincuentes sentenciados se identifican como sentenciados) utilizando datos de Instituto Nacional de Estadística y Geografía (INEGI).

Los datos respecto a cada uno de los niveles académicos utilizados para el análisis se obtuvo de dos fuentes de la Secretaria de Educación Pública, entre ellos los informes anuales respecto a los egresados de cada uno de los niveles educativos y el software IndiSEP del Sistema para el Análisis de la Estadística Educativa (SAEE) y de la Dirección General de Planeación y Estadística Educativa. El nivel educativo de la población fue medido por medio del número de egresados en los niveles educativos de bachillerato y posgrado.

Algo importante en esta investigación, es conocer el efecto de cada uno de los niveles educativos sobre la IED, para poder realizar el objetivo, se realizaron dos modelos, en los cuales las variables inflación, ingreso per cápita, inversión en infraestructura carretera y portuaria, suscripciones de telefonía local fija y delincuencia, permanecerán en cada uno de los dos modelos, pero los niveles educativos serán modificados en cada uno de los modelos por el nivel bachillerato y el nivel posgrado en el segundo modelo.

A continuación se muestran las ecuaciones correspondientes a cada uno de los modelos explicados.

En el modelo 1; IED $_{\text {it }}=a+b_{11 \text { NFLit }}+b_{\text {2IPERCAPit }}+$ $b_{\text {3INFCARRit }}+b_{4 I N F P O R T i t}+b_{\text {5SUSCTELit }}+b_{6 \text { DELINCit }}+$ $\mathrm{b}_{7 \mathrm{BACHit}}+$ ?

En el modelo 2; IED $_{\text {it }}=a+b_{1 \text { INFLit }}+b_{\text {2IPERCAPit }}+$ $b_{\text {3INFCARRit }}+b_{4 \text { INFPORTit }}+b_{\text {5SUSCTELit }}+b_{6 \text { DELINCit }}+$ $\mathrm{b}_{8 \mathrm{POSit}}+$ ?

Donde IED es la Inversión extranjera directa medida en miles de dólares; a es el valor de la constante o la intercepción de la curva con el eje de ordenadas; la inflación durante el periodo de análisis se representa como $b_{1 \mathrm{NFLit}}$; el ingreso per cápita es $b_{\text {2IPERCAPit }}$; la inversión en infraestructura carretera está representada como $b_{3 \text { INFCARRit }}$; la inversión en infraestructura portuaria es $\mathrm{b}_{4 \text { INFPORTit? }}$; el número de suscripciones a líneas telefónicas fijas $b_{\text {5SUSCTELit }}$; los delincuentes procesados y sentenciados por fuero federal y fuero común $\mathrm{b}_{\text {6DELINCit }}$.

Para representar los niveles educativos, el número anual de egresados de nivel bachillerato es $b_{7 \mathrm{BACH}}$ y el número anual de egresados de posgrado $\mathrm{b}_{8 \mathrm{Pos}}$; por último, ? es igual al error estocástico o bien todas las demás variables que podrían influir en la IED y que no están dentro del modelo. Todos los datos durante el periodo $1994-2013$.

El procesamiento y análisis de los datos se realizó con la ayuda del software SPSS versión 23. El resultado de la regresión lineal múltiple será medido a través del coeficiente de determinación, mismo que representa la bondad del ajuste de la 
regresión, es decir, qué tan bien se ajusta la línea de regresión al conjunto de observaciones expresados por la manera en que las variables de inversión en infraestructura y educación explican la IED.

Este método pretende que las diferencias entre observaciones y la línea de regresión sean lo más pequeñas posibles. Por lo tanto, expresa en qué medida la variable IED es explicada por la inflación y el ingreso per cápita, la inversión en infraestructura carretera y portuaria, las suscripciones a líneas telefónicas fijas, la delincuencia y el nivel educativo.

Se medirá en qué porcentaje explican las variables el modelo utilizando el coeficiente de determinación o de la bondad del ajuste $\mathrm{R}^{2}$, así como el valor $p$ de significancia de cada una de las variables en cada uno de los modelos.

\section{ANÁLISIS Y RESULTADOS}

Relación X y Y de la variable dependiente con cada una de las variables independientes.

Para comenzar en análisis, se realizó la estimación de la curva de regresión para cada una de las variables de educación usadas en los modelos.

Se observa la relación existente entre la variable dependiente IED y la variable independiente número de egresados de bachillerato, se observa una relación directa, que expresa que a mayor número de egresados, mayor IED, esto quiere decir, que cada vez que el número de egresados aumenta en una unidad, la IED aumenta en 0.022 unidades. Así mismo, muestra que cuando el número de egresados de posgrado aumenta en una unidad, la IED aumenta también, en 0.200 unidades.

Como se comentó en la revisión de la literatura, se considera que la educación es una determinante del crecimiento de una economía, era algo de esperar en el análisis encontrar un efecto positivo que muestra que a mayor personas egresadas en los niveles educativos, mayor IED.

\section{Estimación de la inversión Extranjera Directa a través de dos modelos}

Posteriormente, se realizó el análisis de regresión lineal múltiple.

En cada uno de los modelos las variables tienen significancia estadística, algo interesante en estos resultados y que difiere del análisis de las variables de manera individual, es el efecto de cada una de las variables en el modelo, respecto a cuándo interactúan conjuntamente con las demás variables que también se consideran determinantes de la IED.

Analizando las variables independientes, en el modelo número uno y en el modelo dos el valor del impacto que la inflación tiene sobre la IED es negativo, lo cual parece muy razonable, dado que a mayor inflación es menos deseable invertir en un país, pues podría afectar negativamente los rendimientos de la inversión, hablando de valores nominales. El ingreso per cápita, es una variable que tiene un efecto positivo sobre la inversión, lo cual es congruente con los resultados de (Gaviria y Gutiérrez, 1993).

Pertinente a lo mencionado por Nourzad, Greenwold y Yang (2014), respecto a cómo la infraestructura ayuda a mejorar los efectos de la IED (p. 210); la inversión carretera e inversión portuaria obtienen un efecto positivo sobre la IED debido a que parte del transporte de mercancías se realiza a través de vías marítimas y terrestres, la inversión que el país anfitrión realice en este medio de transporte incentivará a la IED.

Las suscripciones telefónicas tienen un efecto negativo sobre la inversión extranjera directa, pero solo para el primer modelo en el cual es significativo al $5 \%$, mientras que para el segundo modelo no lo es, lo cual puede deberse al 
desarrollo de otro tipo de tecnología en comunicaciones. La delincuencia tiene un efecto significativo y positivo en ambos modelos sobre la IED. En los dos últimos resultados mencionados, es necesario tomar en cuenta que estas variables explicativas pueden ser sensibles a los efectos de demás variables independientes del modelo.

Por lo tanto, aunque podría suponerse que el número de suscripciones telefónicas puede tener un efecto positivo sobre la IED y suponerse que la delincuencia tiene un efecto negativo sobre la IED, el efecto para ambas fue inverso a lo esperado y a lo mostrado en la relación individual de cada variable independiente con la variable dependiente, tomando en cuenta el efecto que las demás variables causan en el modelo.

Analizando detalladamente cada uno de los parámetros de educación estimados en los dos modelos de regresión, se observó que los parámetros representa un impacto negativo sobre la IED. La proporción del impacto de la educación a nivel bachillerato se explica en el primer modelo, en el cual la variable bachillerato es significativa a un nivel de $1 \%$ y muestra que cuando el número de egresados de bachillerato aumenta en una unidad, la IED se reduce en 0.056 unidades. La educación a nivel posgrado se explica en el segundo modelo, en el que la variable posgrado es significativa a un nivel del $5 \%$ y explica que cuando el número de egresados de posgrado aumenta en una unidad, la IED se reduce en 1.007 unidades.

Con base en estos resultados, puede decirse que un alto nivel de estudios afecta negativamente la IED, y a niveles más altos de estudios este impacto es más desfavorable, dada la hipótesis de que un alto nivel educativo no es requerido.

La relación con la IED puede ocurrir de la siguiente forma: a mayor número de egresados del nivel educativo, menor IED, esto debido a que con la llegada de nuevas empresas para las cuales se requiere un mayor número de personal para trabajar, personal que como se mencionó con anterioridad puede no requerir instrucción o formación alguna, y un alto nivel educativo afecta que se pueda emplear la mano de obra con pocas habilidades como la que requieren las EMN.

Adicionalmente, es posible tener una respuesta del por qué el bachillerato tiene una repercusión menor en este efecto negativo que causa la educación; a menor nivel de instrucción estas personas se emplearan en los puestos que son requeridos, mientras que quienes tienen una mayor formación académica pueden no ser requeridos para estas tareas, o en ciertos casos no querer realizar esos trabajos, para los cuales están sobre calificados.

Los resultados obtenidos se alinean con lo obtenido por (Liu, Lou, Qiu, y Zhang, 2014), al encontrar que la IED al crear empleos con mano de obra no calificada, afecta la decisión de las personas acerca de los estudios superiores, por lo que a largo plazo se evita la acumulación de capital humano (p.104).

La ecuación de cada uno de los modelos de regresión lineal múltiple, es la siguiente:

$$
\mathrm{IED}_{\text {MODELO1 }}=-252850.63-1657.178_{\mathrm{INFL}}+
$$
$34.174_{\text {PERCAP }}+0.198_{\text {INFCARR }}+0.925_{\text {INFPORT }}-$ $4.919_{\text {SUSCTEL }}+0.515_{\text {DELINC }}-0.056_{\text {BACH }}+$ ?

$$
\begin{aligned}
& \text { IED }_{\text {MODELO2 }}=-289049.62-2381.911_{\text {INFL }}+ \\
& 35.17_{\text {IPERCAP }}+0.342_{\text {INFCARR }}+1.369_{\text {INFPORT }}- \\
& 5.287_{\text {SUSCTEL }}+0.618_{\text {DELINC }}-1.007_{\text {POS }}+?
\end{aligned}
$$

Por lo tanto, las ecuaciones nos sirven para realizar un pronósticos de los valores de la IED; es decir, se podría realizar un pronóstico del efecto sobre la IED con niveles dados que las variables independientes podrían tener.

Finalmente, la herramienta que mide la bondad

${ }^{2}$ ajustado, muestra en qué porcentaje un modelo puede ser explicado por las variables 
utilizadas. Esto quiere decir, que en el Modelo 1, las variables utilizadas explican el $97 \%$ de la IED; el Modelo 2, explica el $91.7 \%$ de la IED.

\section{CONCLUSIONES}

Al realizar esta investigación, se pretendía conocer y analizar la relación existente entre la IED y el nivel educativo, así como algunas otras variables consideradas pertinentes para incentivar o desincentivar la IED.

Los primeros resultados sugieren que la infraestructura portuaria y la infraestructura carretera benefician de manera positiva la IED, si para realizar la interpretación del análisis nos sustentamos con la revisión previa de la literatura, lo cual se justifica debido a que la infraestructura de transporte es necesaria para los negocios y atrae la IED; mientras que la inflación tiene un efecto negativo debido a que con la inflación se reduce el poder adquisitivo de las personas y podría reducir los rendimientos futuros de los inversionistas.

El nivel educativo, el cual es el punto principal de esta investigación, se encuentra relacionado de manera inversa con la IED, un mayor nivel educativo afecta en mayor medida la inversión extranjera directa, porque a medida que existan más inversión extranjera en el país se requerirán menos personal con alto nivel educativo.

Esto a su vez llama a la deserción escolar debido a que quienes estudian preferirán ocuparse que continuar estudiando, quienes estudiaron y obtuvieron altos niveles educativos no podrán emplearse al no ser necesarios, precisamente por los pocos requerimientos al emplear personal y al mínimo nivel de transferencia de tecnología de las EMN.

Al haber entrada de inversión extranjera directa, existirá empleo para quienes tienen un nivel menor de formación, lo cual explica los niveles menores en las tasas de desempleo que existe para los egresados de menores niveles académicos.

Sería adecuado que para próximas investigaciones retomar el tema de la educación y la transferencia de tecnología, además de profundizar en el efecto del desempleo y el nivel educativo para analizar los resultados al utilizar un mayor número de variables a los modelos que expliquen la inversión extranjera directa.

\section{REFERENCIAS}

Centro de Estudios de Finanzas Públicas. (2014). Inversión Extranjera Directa por Entidad Federativa. Recuperado de http:// www.cefp.gob.mx/intr/bancosdeinformacion/ estatales/.../is020.xls

De la Cruz Gallegos, J. L., Canfield Rivera, C., y González Castro, P. (2009). Economic growth, foreign direct investment and international trade: Evidence on causality in the mexican economy. (Universa, Ed.). Revista Brasileña de Economía de Empresas, 9(1), 18. ISSN: 1676-8000 (electrónico)

Dutrénit, G., y Vera-Cruz, A. O. (2005). Technological capability accumulation in the 'maquila industry' in Mexico. Cuadernos EBAPE.BR, 3,2. doi: 10.1590/S167939512005000500016

Gaviria, A., y Gutiérrez, J. A. (1993). Inversión extranjera directa y crecimiento económico. Archivos de Macroeconomía, 22.

INEGI. (2015). Registros administrativos judiciales en materia penal. Recuperado de

http://www.inegi.org.mx/est/lista_cubos/ consulta.asp $x ? \mathrm{p}=\mathrm{adm} \& \mathrm{c}=10$

Jordaan, J. A. (2008). State caracteristics and the locational choice of Foreign Direct Investment: Evidence from Regional FDI in Mexico 19892006. Growth and Change, 39(3), 389-413. doi: 10.1111/j.1468-2257.2008.00431.x

Kar, S. (2013). Exploring the causal link between FDI and human capital development in India. Decision, 4(1), 3-13. doi: 10.1007/s40622013-0001-5

Kato-Vidal, E. L. (2013). Foreign investment and wages: a crowding out effect in Mexico. 
Latin America Journal of Economics, 50(2), 209231. doi: 10.7764/LAJE.50.2.209

Keller, K. R. (2005). Investment primary, secondary and higher education and the effects on economic growth. Contemporary Economic Policy, 24(1), 18-34. doi: 10.1093/ cep/byj012

Kumaran, G. B. (2008). Role of multinational corporations in automobile industries: A comparative study betwen India and Mexico. Revista Mexicana de Estudios sobre la Cuenca del Pácifico, 2(3), 131-163. ISSN: 1870-6800 (electrónico)

Kunhardt, B., y Gutiérrez-Haces , M. T. (2013). Localización geográfica y sectores de inversión: Factores decisivos en el desempeño de las multinacionales mexicanas durante la crisis. Journal of Economics, Finance and Administrative Science, 18(34), 34-44. doi:10.1016/S20771886(13)70021-0

Liu, X., Lou, Y., Qiu, Z., y Zhang, R. (2014). FDI and economic development: Evidence from China's regional growth. Emerging Markets Finance and Trade, 50(6), 81-106. doi: 10.1080/1540496X.2014.1013852

Molina-Martínez, R., y Alcaraz-Vargas, J. L. (2012). The competition of mexican states:

Attracting foreign direct investment. Advences in Competitiveness Research, 20, 59-74. ISSN: 1077-0097 (electrónico)

Moreno, N. A., y Méndez, C. Á. (2014). Análisis prospectivo de las políticas públicas de transferencia de tecnología en México. Estrategias Tecnológicas,1-7. Recuperado de http://upaep.mx/micrositios/coloquios/ coloquio $2014 / \mathrm{m}$ e moria s/

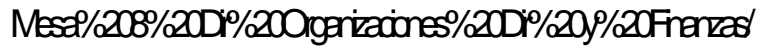
Organizaciones/a1\%20Nelson.pdf

Neycheva, M. (2013). Does higher level of education of the labor force cause growth?

Evidence from Bulgaria. Economic Change and Restructuring, (46), 321-339. doi 10.1007/ s10644-013-9139-6

Nourzad, F., Greenwold, D. N., y Yang, R. (2014). The interaction between FDI and infrastructure capital in the development process. International Advances In Economic Research, 20(2), 203-212. doi: 10.1007/ s11294-013-9457-5
Nuñez, I., y Livanos, I. (2010). Higher education and unemplyment in Europe: An analysis of the academic subject and national effects. Springer Science+Business Media (59), 475487. doi: 10.1007/s10734-009-9260-7

OECD. (2014). Country Note: Education at a glance 2014: OECD Indicators. Recuperado de http://www.oecd.org/edu/MexicoEAG2014-Country-Note.pdf

OECD. (2013). Country note: Mexico. Recuperado de http://www.oecd.org/edu/ Mexico_EAG2013\%20Country\%20Note.pdf

Ramos, G. (mayo de 2015). México. Policy priorities to upgrade the sills and knowledge of mexicans for greater productivity and innovation. Recuperado de http:// www.oecd.org/mexico/mexico-policypriorities-to-upgrade-skills-and-knowledge-ofmexicans.pdf

Ruiz, P., y Ordaz, J. L. (2013). Evolución reciente del empleo y el desempleo en México. Economía Unam, 8(23), 91-105. ISSN: 1665952X (Impreso).

Secretaría de Comunicaciones y Transportes. (2014). Principales estadísticas de comunicaciones y Transportes. Recuperado de http://www.sct.gob.mx/informaciongeneral/planeacion/estadistica/principalesestadisticas-del-sector.

Sistema de Análisis de la Estadística Educativa (Version 1) [Software de computación]. México: Indisep.

Spieb, K. C. (2013). Investments in education: The early years Offer great potential. DIW Economic Bulletin,3(10),1-9. Recuperado de https://www.diw.de/documents/publikationen/ 73/diw_01.c.429357.de/diw_econ_bull_201310-1.pdf

Waldkirch, A., Nunnenkamp, P., y Alatorre Bremont, J. E. (2009). Employment effects of FDI in Mexico's non-maquiladora manufacturing. Journal of Development Studies, 45 (7), 1165-1183. doi:10.1080/ 00220380902952340

Young, S., y Lan, P. (1997). Technology transfer to China through foreign direct investment. Regional Studies, 31(7), 669-679. doi: 10.1080/003434097 